\title{
Manejo del prurito durante el embarazo
}

\author{
J. I. SÁnChez-MÉndez, M. J . López-RodRíGuez* \\ Doctor en Medicina y Cirugía. Universidad Autónoma de Madrid. Especialista en \\ Obstetricia y Ginecología. Servicio de Ginecología. Hospital La Paz. Madrid. \\ * Licenciada en M edicina y Cirugía. U niversidad de Santiago de Compostela. \\ Especialista en Obstetricia y Ginecología. Servido de Ginecología. \\ Hospital Ramón y Cajal. Madrid.
}

\section{Pruritus during pregnancy: a practical approach}

\section{RESUMEN}

El prurito, un síntoma frecuente durante la ges tación, puede ser manifestación de un gran número de enfermedades. Si nos centramos en aquellas propias del embarazo podemos distinguir dos grandes grupos. Uno en el que no hay lesiones dermatológicas propias, formado por la colestasis intrahepática del embarazo, y su forma menor el prurito gestacional. Y otro más amplio y eminente mente dermatológico, caracterizado por la apari ción de diversas lesiones en la piel. El tratamiento sintomático inicial es común para todos los proce sos, y consiste en la administración de antiprurigi nosos tópicos.

En el presente trabajo enfocamos este síntoma desde una perspectiva eminentemente práctica que permite de forma sencilla su diagnóstico diferen cial, manejo, y orientación terapéutica inicial.

Palabras clave: Prurito. Colestasis intrahepáti ca. Herpes gestationis. Antipruriginosos.

\section{INTRODUCCIÓN}

El prurito es un síntoma frecuente durante el embarazo ya que aproximadamente entre el 3 y el $15 \%$ de las gestantes se ven afectadas por él al menos en algún momento ${ }^{1,2}$. Las causas que lo pueden producir son muchas, pero a grandes rasgos se dividen en dos grupos:

-Enfermedades propias, aunque no exclusivas, del embarazo.

-Enfermedades coexistentes con el embarazo $(\text { Tabla I })^{3}$.

\section{ABSTRACT}

Pruritus, a frequent symptom during pregnancy, can be present in a large number of diseases. If we select those characteristic of the pregnancy, we can difference two groups. The first one, without typical skin lesions, includes the intrahepatic cho lestasis and its minor form, the gestational pruri tus. The other one, wider and eminently dermatolo gical, is characterized by the appearance of diverse lesions in the skin. The initial symptomatic treatment is common for all the processes, and it consists on the administration of topical antipruri tus substances.

In this paper we approach this symptom from an eminently practical perspective that allows, in a simple way, the differential diagnosis, and the ini tial treatment.

Key words: Pruritus. Intrahepatic cholestasis. Herpes gestationis. Antipruritus substances.

En este trabajo vamos a centrarnos principalmente en las primeras, en las que el prurito suele ser el motivo de consulta, y para cuyo diagnóstico diferencial propondremos la aplicación de un sencillo algoritmo.

\section{CUADROS CLÍNICOS}

\section{Prurito gravídico (PG)}

Es una forma leve de colestasis del embarazo descrita por primera vez por Keher, en $1907^{4}$. Su 


\begin{tabular}{|l|}
\hline ENFERMEDADES QUE PUEDEN CURSAR CON PRURITO \\
\hline - Candidiasis \\
- Trichomonas \\
- Pediculosis \\
- Sarna \\
\hline - Hepatitis vírica \\
- Hepatitis tóxica \\
- Litiasis biliar \\
- Hemólisis \\
- Ictericia fenotiacínica \\
- Degeneración grasa aguda del hígado \\
\hline - Linfoma de Hodgkin, cáncer de mama, pulmón, \\
- tumor cerebral \\
- Mieloma múltiple \\
- Policitemia vera \\
- Enfermedad de Sjogren \\
- Esclerosis múltiple \\
- Hiper/ hipotiroidismo \\
- Sarcoidosis \\
- Neuropatía crónica \\
\hline - Patología psiquiátrica \\
\end{tabular}

incidencia oscila entre el 0,02 y el $2,4 \%$ de las gestaciones ${ }^{5}$. Suele aparecer en el tercer trimestre y regresar a la normalidad en el puerperio. Tiene tendencia a recidivar en embarazos subsiguientes así como con la toma de contraceptivos ${ }^{5}$. El pronóstico, tanto materno como fetal es bueno.

El diagnóstico es fundamentalmente clínico, y casi de exclusión. Se caracteriza por la presencia de prurito nocturno, sobre todo palmo-plantar, sin lesión dermatológica aparente, salvo ocasionales lesiones de rascado. Algunos autores han descrito hiperemesis con mayor frecuencia y duración, así como "dispepsia biliar".

Los datos de laboratorio pueden ser normales, o como mucho encontrarse un aumento moderado de la fosfatasa alcalina, la bilirrubina y las transaminasas, pero siempre en los límites de la normalidad. Si estos valores están claramente elevados deberemos pensar que nos hallamos ante una colestasis intrahepática del embarazo.

El tratamiento es inicialmente sintomático (antipruriginosos tópicos) ayudado por una dieta en la que las grasas sean predominantemente polinsaturadas. En los casos más graves se añadirá colestiramina y, si no resulta eficaz, fenobarbital a dosis de 60-120 mg/día. Otra alternativa es la administración de prednisona (10-15 mg/día). El empleo de luz ultravioleta puede ser una buena opción, pero aún está poco contrastada ${ }^{4}$.

\section{Colestasis intrahepática del embarazo (CIE)}

Es una enfermedad con un claro componente genético que se presenta con gran frecuencia entre las gestantes escandinavas y aquéllas procedentes de una tribu chilena. También se ha apuntado a un déficit de selenio en su desencadenamiento ${ }^{6}$, así como a la administración exógena de progesterona ${ }^{7}$.

La clínica es similar al PG aunque de mayor intensidad. Puede existir ictericia pero va precedida siempre de un intenso prurito. De hecho la CIE constituye la segunda causa de ictericia durante la gestación.

Para poder pensar en ella se requiere descartar primero la toma de fármacos capaces de producir colestasis (Tabla II), y la posible existencia de una colestasis extrahepática.

Está descrita una mayor frecuencia de prematuridad y de bajo-peso, así como de hemorragias postparto.

Entre los datos del laboratorio destacan:

-Alteración de la bilirrubina y las enzimas hepáticas: se observa una hiperbilirrubinemia, fundamentalmente conjugada y que no suele ser mayor de $5 \mathrm{mg} / \mathrm{ml}$, un aumento de las fosfatasas alcalinas (que no llegan a triplicarse, mientras que en las colestasis extrahepáticas sí), un aumento de la 5' Nucleotidasa y de la $\gamma$ glutamil transpeptidasa, etc.

- La síntesis de ácidos biliares es menor y cualitativamente distinta: el principal ácido biliar en estos pacientes es el ácido cólico, en lugar del quenodesoxicólico. Así para el diagnóstico precoz de la CIE, como para su seguimiento, se han pro-

\begin{tabular}{l} 
Tabla II \\
\hline \multicolumn{1}{c|}{ FÁRMACOS QUE PUEDEN PRODUCIR PRURITO } \\
\hline \multicolumn{1}{c|}{ PRODUCTORES DE COLESTASIS } \\
- Estrógenos \\
- Clorpromacina \\
- Estolato de eritromicina \\
POTROS MECANISMOS \\
- Alfa metildopa, \\
- Sulfamidas \\
- Metoclopramida \\
- Quinidina \\
- Mórficos
\end{tabular}


puesto como mejores marcadores la existencia de una concentración de ácidos biliares totales en plasma mayor de $11 \mu \mathrm{mol} / 1$ con una relación ácido cólico/quenodesoxicólico superior a 1,5 con un porcentaje de ácido cólico superior al $42 \%$.

A nivel molecular se cree que estos pacientes tienen un defecto selectivo en la secreción de metabolitos sulfatados de la progesterona hacia la bilis, causado por una alteración genética en el transportador canalicular de esteroides sulfatados y en especial disulfatados, y/o en su regulación, y que se pondría de manifiesto ante la presencia de determinados metabolitos estrogénicos ${ }^{9,10}$.

Si se practica una biopsia hepática, lo cual no está indicado de forma rutinaria, se observan, en una citarquitectura conservada, pigmentos biliares intra y extracelulares así como una hiperplasia de las células de Kupffer.

Persiste hasta el postparto, y su evolución es insidiosa: primero desaparece el prurito y luego, en un plazo que no suele ser superior a las dos semanas, la ictericia. La fosfatasa alcalina tarda unos 3 meses en normalizarse.

Tiende a recidivar en embarazos sucesivos y parece predisponer al desarrollo de una litiasis biliar posterior.

El tratamiento básico es igual que el del PG aunque suele ser necesaria casi siempre la administración de colestiramina. Recientemente se ha propuesto la S-adenil-metionina (SAME) como alternativa terapéutica ${ }^{11}$, si bien el fármaco más prometedor, y con el que más estudios se están realizando, es el ácido ursodesoxicólico ${ }^{12}$. En los casos rebeldes será preciso el empleo de fenobarbital y/o prednisona por vía oral.

Además, es preciso estrechar la vigilancia fetal mediante la realización de perfiles biofísicos (ecografía, monitorización cardiotocográfica, etc.) periódicos. Algunos autores recomiendan que alcanzada la viabilidad fetal se induzca el parto.

\section{Prurigo gestationis (PgG)}

Descrito por Besnier en $1904^{4}$, se caracteriza por la aparición de pequeñas pápulas (1-2 mm), muy pruriginosas, localizadas de forma simétrica, preferentemente en las extremidades inferiores. También pueden extenderse por las superficies de extensión de los brazos, el dorso de las manos, muslos, piernas y el dorso de los pies. En los casos graves se pueden afectar los hombros, la región escapular y el tórax.

Se intensifica a lo largo del embarazo, y remite rápidamente tras el parto dejando tan sólo una ligera pigmentación. Puede recidivar en un nuevo embarazo.
No se detectan alteraciones analíticas hepáticas, y no produce efectos adversos en la madre ni en el feto.

El tratamiento es sintomático empleando para ello corticoides tópicos.

\section{Herpes Gestationis (HG)}

Descrito por primera vez por Milton en $1872^{4}$, es una erupción vesículo-ampollosa que cursa con un prurito muy intenso. Puede aparecer en cualquier momento del embarazo pero lo más frecuente es que lo haga en el segundo trimestre. Afecta a una de cada 10.000 embarazadas.

La erupción, que viene precedida por unos pródromos de fiebre y cefalea, se extiende generalmente de forma simétrica por el tronco (periumbilical) y las extremidades, y suele respetar a las mucosas. Está formada por unas placas eritematosas, muy pruriginosas y recidivantes, que evolucionan a vesículas tensas, grandes, transparentes, de aspecto penfigoide y contenido serohemorrágico. Son resistentes pero acaban por romperse y dar lugar a una erosión en la piel, que después se transforma en costra. Curan sin dejar cicatriz o a lo sumo una zona de hiperpigmentación.

En el hemograma se encuentra leucocitosis y eosinofilia. Tiene una característica inmunopatológica patognomónica que es la presencia del factor 3 del complemento en el límite dermo-epidérmico del área perilesional ${ }^{13}$.

Suelen curar en la primera semana postparto pero se puede prolongar durante mucho tiempo, incluso hasta años. Recidiva en siguientes embarazos, con la toma de contraceptivos o incluso de forma catamenial.

En los casos leves el tratamiento es sintomático. Ante un cuadro clínico muy florido el ingreso es obligatorio, y se debe iniciar corticoterapia (1 $\mathrm{mg} / \mathrm{kg}$ peso/día) por vía sistémica. Está descrita una mayor morbimortalidad fetal ${ }^{14}$.

\section{Pápulas y placas urticariformes pruriginosas del embarazo (PPUPE)}

Definida en 1979 por Lawley ${ }^{15}$ probablemente engloba a otros muchos cuadros previamente descritos. Se desconoce su incidencia real pero lo cierto es que en los últimos años ha despertado un gran interés y son muchas las publicaciones sobre la misma $^{16-18}$.

Se caracteriza por la presencia de pápulas urticariformes y placas, que suelen distribuirse de forma simétrica por el tronco y la raíz de las extremidades, respetando la cara. En su patogénesis parece intervenir la sobredistensión abdominal ${ }^{19}$ y por ello típica- 
mente afecta a primigrávidas, en el tercer trimestre del embarazo, y a las gestaciones múltiples.

Las lesiones desaparecen en el puerperio y el proceso no suele recidivar en los siguientes embarazos ni con la toma de contraceptivos. Un tratamiento tópico suele ser suficiente. No se han descrito efectos adversos sobre el feto.

Otras dermatosis pruriginosas del embarazo menos frecuentes son:

-La erupción toxémica del embarazo ${ }^{20}$.

- La dermatitis papular del embarazo ${ }^{21}$.

- La dermatitis progesterónica autoinmune del embarazo $^{22}$.

- La foliculitis pruriginosa del embarazo ${ }^{23}$.

\section{DIAGNÓSTICO DIFERENCIAL}

Para la aproximación diagnóstica de una gestante que consulte por prurito podemos emplear el algoritmo representado en la figura 1 en el que quedan reflejados los principales cuadros clínicos.

\section{TRATAMIENTO}

El tratamiento sintomático es común para todos los procesos. Consiste en la administración de:

-Lociones antipruriginosas. Fundamentalmente se pueden emplear lociones de hidrocortisona al $1 \%$; también se han observado buenos resultados con el empleo de una emulsión de aceite de oliva / agua de limón con mentol.

\begin{tabular}{l}
\hline $\begin{array}{l}\text { Suniderma }{ }^{\circledR} \\
\text { Fórmula magistral }\end{array}$ \\
\hline
\end{tabular}

-Antihistamínicos. Se deben emplear aquéllos que pasen la barrera hematoencefálica: dexclorfeniramina, etc., ya que su acción antipruriginosa se debe fundamentalmente a sus efectos sedantes.

\begin{tabular}{lll}
\hline Polaramine repetabs ${ }^{\circledR}$ & $\begin{array}{l}\text { grageas de } 6 \mathrm{mg} \\
\text { de liberación retard }\end{array}$ & 20 grageas \\
Polaramine tabletas ${ }^{\circledR}$ & $2 \mathrm{mg}$ & 20 grageas \\
\hline
\end{tabular}

-Las benzodiacepinas se han mostrado igual e incluso más eficaces que los antihistamínicos en el control del prurito y para algunos autores son de elección ${ }^{24}$.

\begin{tabular}{lll}
\hline Diazepam 5-10 mg & $5-10 \mathrm{mg}$ & $30-25$ comp. \\
\hline
\end{tabular}

\section{Tratamiento específico para la colestasis y prurito gravídicos}

- Colestiramina. Es una sustancia de elevado peso molecular, que no se absorbe tras su administración por vía oral. Se une a los ácidos biliares en el intestino delgado impidiendo su reabsorción. En general ejerce la misma acción con todas las sustancias de circulación enterohepática.

Se debe administrar disuelta en un vaso de agua u otro líquido (zumo), y no como polvo seco. Aún así resulta molesta de ingerir por su carácter arenoso, y puede producir irritación en la mucosa bucal. Existe una presentación en forma microporosa que se tolera algo mejor. Si hay que administrar algún otro fármaco se hará 1-2 horas antes o 4 horas después, dada la elevada capacidad quelante de la colestiramina. La dosis habitual es de 4 gramos cada 6 a 8 horas; otra pauta de administración es 4 gramos antes y después del desayuno, y 4 gramos antes de la comida y la cena.

Como efectos secundarios puede producir estreñimiento, náuseas, vómitos, hipertriacilgliceridemia y malabsorción de vitaminas liposolubles, calcio, ácido fólico, etc. Por ello, ante tratamientos prolongados se recomiendan suplementos parenterales de vitamina $\mathrm{K}$ (control del tiempo de protrombina), así como de otras vitaminas liposolubles (A y D). Es conveniente administrar también suplementos orales de calcio y de ácido fólico.

\begin{tabular}{llll}
\hline Lismol ${ }^{\circledR}$ & sobres de $4 \mathrm{~g}$ & 50 sobres & \\
Efensol & sobres de $3 \mathrm{~g}$ & 40 sobres & $\begin{array}{l}\text { en forma de resina } \\
\text { microporosa }\end{array}$ \\
\hline
\end{tabular}

-Ácido ursodesoxicólico. En la CIE intervendría como facilitador de la sulfatación de sustancias esteroideas, y por tanto de su eliminación bi$\operatorname{liar}^{25}$.

Se ha estudiado su efecto fetal, y se ha constatado también un incremento de la concentración total de ácidos biliares en el meconio de los hijos de madres que han padecido una CIE, sin que esto sea perjudicial para ellos ${ }^{26}$. Asimismo es seguro durante la lactancia, en caso de que sea preciso su administración ${ }^{27}$.

La dosis recomendada es de 3-5 cápsulas al día repartidas en 2 dosis.

\begin{tabular}{lll}
\hline Ursobilane ${ }^{\circledR}$ & caps. $150 \mathrm{mg}$ & 60 cápsulas \\
Ursolitel ${ }^{\circledR}$ & caps. $150 \mathrm{mg}$ & 60 cápsulas \\
\hline
\end{tabular}




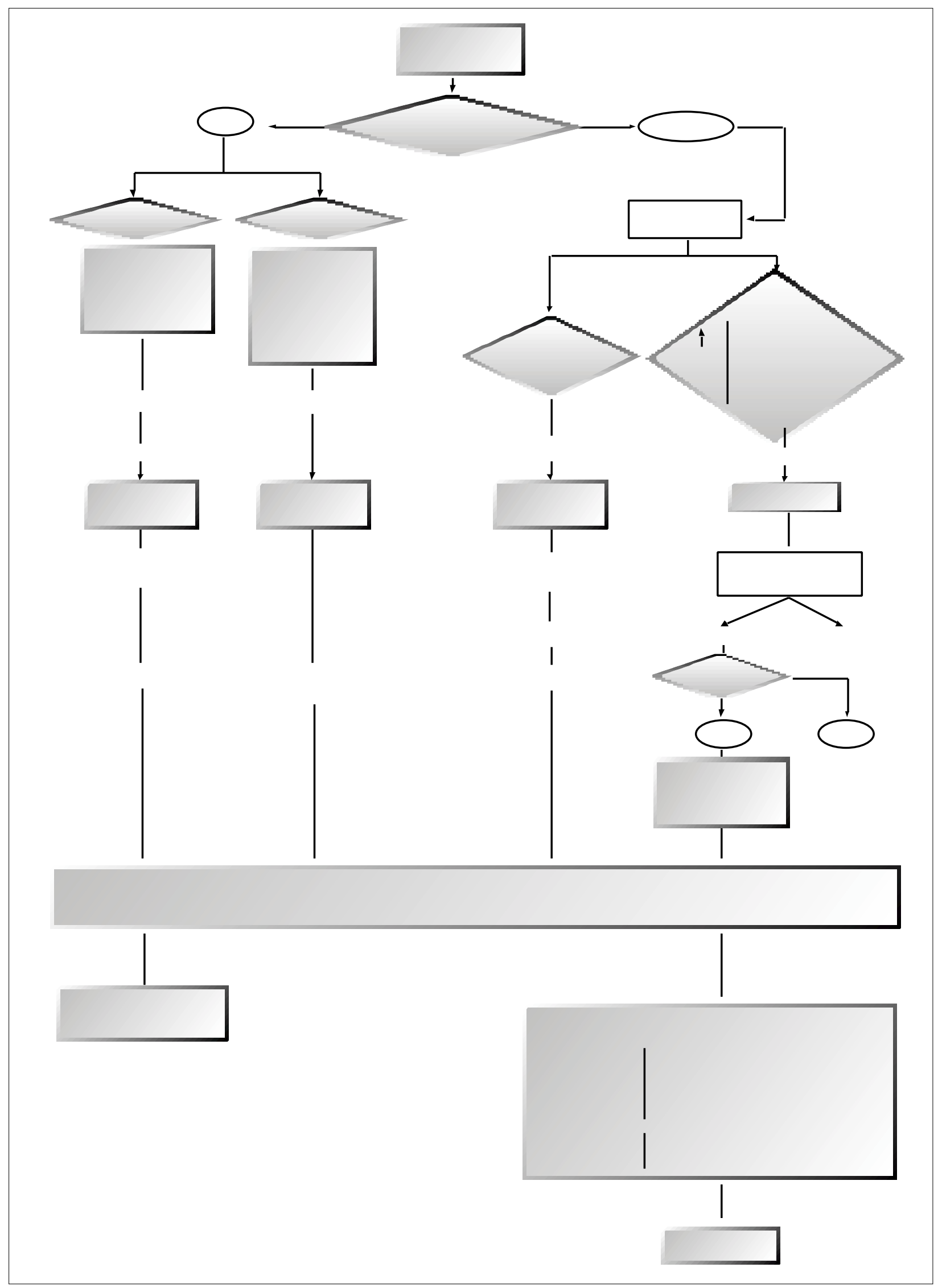

Figura 1

Algoritmo para el diagnóstico diferencial. 
-S. adenil metionina. La sulfoadenosil L metionina es un donador de metilos activos que participa en todas las reacciones de transmetilación del organismo, excepto las de su resíntesis. Además su metabolización produce un potente agente antioxidante como es el glutation. Se administra por vía parenteral a dosis de $200 \mathrm{mg}$ cada 12/24 horas, si bien se puede aumentar hasta un máximo de $800 \mathrm{mg}$ al día.

\begin{tabular}{lll}
\hline S-Amet 200 & $200 \mathrm{mg}$ & $5 \mathrm{amp}$. \\
\hline
\end{tabular}

- Corticoterapia. Una utilización juiciosa de corticoesteroides durante la gestación, tanto de forma tópica como sistémica, no ha mostrado ser dañina para el feto.

Prednisona (10-15 mg/día).

\begin{tabular}{lll}
\hline Dacortin ${ }^{\circledR}$ & $5 \mathrm{mg}$ & $30-60$ comp. \\
Prednisona Alonga & 5 y $10 \mathrm{mg}$ & $30-60 / 30$ comp. \\
\hline
\end{tabular}

-Fenobarbital. Es eficaz en los casos rebeldes. Se emplea a dosis de 50-100 mg/día.

\begin{tabular}{lll}
\hline Luminal & $100 \mathrm{mg}$ & 50 comp. \\
Gardenal & $50 \mathrm{mg}$ & 30 comp. \\
\hline
\end{tabular}

\section{CORRESPONDENCIA:}

J. I. Sánchez-Méndez

Servicio de Ginecología

Hospital La Paz

Paseo de la Castellana, 261

28046 Madrid

\section{Bibliografía}

1. Dacus JV, Muram D. Pruritus in pregnancy. South Med J 1987; 80: 614-7.

2. Fahmer L, Murray JC. The skin. Principles of Medical Therapy in Pregnancy. Plenum Publishing Corporation 1986; 172: 1298-314.

3. Dacus JV. Prurito durante le embarazo. Clínicas Obstétricas y Ginecológicas 1990; 4: 715-21.

4. Noguera Tusquets X. Dermopatías durante la gestación. Jano 1989; 36: 93-8.

5. Johnston WG, Baskett TF. Obstetric cholestasis: a 14year review. Am J Obstet Gynecol 1979; 133: 299-301.

6. Reyes H. J Review: intrahepatic cholestasis. A puzzling disorder of pregnancy. Gastroenterol Hepatol 1997; 12 (3): 211-6.

7. Bacq Y. Intrahepatic cholestasis in pregnancy. The hepatologist's point of view. J Gynecol Obstet Biol Reprod 1993; 22: 533-8.

8. Brites D, Rodrigues CM, van-Zeller H, Brito A, Silva R. Relevance of serum bile acid profile in the diagnosis of intrahepatic cholestasis of pregnancy in an high incidence area: Portugal. Eur J Obstet Gynecol Reprod Biol 1998; 80: 31-8.

9. Reyes H, Sjovall J. Bile acids and progesterone metabolites in intrahepatic cholestasis of pregnancy. Ann Med 2000; 32: 94-106.

10. Meng LJ, Reyes H, Palma J, Hernández I, Ribalta J, Sjovall J. Profiles of bile acids and progesterone metabolites in the urine and serum of women with intrahepatic cho- lestasis of pregnancy. J Hepatol 1997; 27: 346-57.

11. Bonessio L, Ciardo A, Spina V, Morini A. Intrahepatic cholestasis in pregnancy. Clin Ter 1996;147: 377-84.

12. Floreani A, Paternoster D, Melis A, Grella PV. S-adenosylmethionine versus ursodeoxycholic acid in the treatment of intrahepatic cholestasis of pregnancy: preliminary results of a controlled trial. Eur J Obstet Gynecol Reprod Biol 1996; 67: 109-13.

13. Foidart JM, Yaar M, Hall R, Gaspard U. Immunopathologic and clinical studies in herpes gestationis. Br J Obstet Gynecol 1981; 88: 153-9.

14. Lawley TJ, Stingl G, Katz SI. Fetal and maternal risk factors in herpes gestationis. Arch Dermatol 1978; 114: 552-5.

15. Lawley TJ, Herz KC, Wade TR. Pruritic urticarial papules and plaques of pregnancy. JAMA 1979; 241: 1696.

16. Alcalay J, Wolf JE Jr. Pruritic urticarial papules and plaques of pregnancy: the enigma and the confusion (editorial). J Am Acad Dermatol 1988; 19: 1115-6.

17. Barrenetxea G, Melchor JC, Barbazán MJ, Aranguren G, Rodríguez-Escudero FJ. Pruritic urticarial papules and plaques of pregnancy. Int J Gynaecol Obstet 1990; 33: 69-72.

18. Alcalay J, Ingber A, Kafri B, Segal J, Kaufmann H, Hazaz B, et al. Hormonal evaluation and autoimmune background in pruritic urticarial papules and plaques of pregnancy. Am J Obstet Gynecol 1988; 158: 417-20.

19. Cohen LM, Capeless EL, Krusinski PA, Maloney ME. Pruritic urticarial papules and plaques of pregnancy and 
its relationship to maternal fetal weight gain and twin pregnancy. Arch Dermatol 1989; 125: 1534-6.

20. Bourne G. Toxemic rash of pregnancy. Proc R Soc Med 1962; 55: 462-4.

21. Spangler AS, Reddy W, Bardwail WA, Emerson K. Papular dermatitis of pregnancy, a new clinical entity? JAMA 1962; 181: 577-81.

22. Bierman SM. Autoimmune progesterone dermatitis of pregnancy. Arch Dermatol 1973; 107: 896-901.

23. Zoberman E, Farmer E. Pruritic folliculitis of pregnancy. Arch Dermatol 1981; 117: 20.

24. Krause L, Shuster S. Mechanism of action of antiemetic drugs. Br Med J 1983; 287: 1199-200.
25. Meng LJ, Reyes H, Axelson M, Palma J, Hernández I, Ribalta J, et al. Progesterone metabolites and bile acids in serum of patients with intrahepatic cholestasis of pregnancy: effect of ursodeoxycholic acid therapy. Hepatology 1997; 26: 1573-9.

26. Rodrigues CM, Marin JJ, Brites D. Bile acid patterns in meconium are influenced by cholestasis of pregnancy and not altered by ursodeoxycholic acid treatment. Gut 1999; 45: 446-52.

27. Brites D, Rodrigues CM. Elevated levels of bile acids in calostrum of patients with cholestasis of pregnancy are decreased following ursodeoxycholic acid therapy. J Hepatol 1998; 29: 743-51. 